Widefield ScIENCE AND TeChNOLOGY For the SKA

SKADS CONFERENCE 2009

S.A. Torchinsky, A. van Ardenne, T. van den Brink-Havinga, A.J.J. van Es, A.J. Faulkner (eds.)

4-6 November 2009, Château de Limelette, Belgium

\title{
Wide Field Polarimetry and Cosmic Magnetism
}

\author{
R. Beck
}

\begin{abstract}
Max-Planck-Institut für Radioastronomie, Auf dem Hügel 69, 53121 Bonn, Germany *
\end{abstract}
\begin{abstract}
The SKA and its precursors will open a new era in the observation of cosmic magnetic fields and help to understand their origin. In the SKADS polarization simulation project, maps of polarized intensity and RM of the Milky Way, galaxies and halos of galaxy clusters were constructed, and the possibilities to measure the evolution of magnetic fields in these objects were investigated. The SKA will map interstellar magnetic fields in nearby galaxies and intracluster fields in nearby clusters in unprecedented detail. All-sky surveys of Faraday rotation measures (RM) towards a dense grid of polarized background sources with the SKA and ASKAP (POSSUM) are dedicated to measure magnetic fields in distant intervening galaxies, cluster halos and intergalactic filaments, and will be used to model the overall structure and strength of the magnetic fields in the Milky Way and beyond. Simple patterns of regular fields in galaxies or cluster relics can be recognized to about $100 \mathrm{Mpc}$ distance, ordered fields in unresolved galaxies or cluster relics to redshifts of $z \simeq 0.5$, turbulent fields in starburst galaxies or cluster halos to $z \simeq 3$, and regular fields in intervening galaxies towards QSOs to $z \simeq 5$.
\end{abstract}

\section{Origin of magnetic fields}

The origin of the first magnetic fields in the early Universe is still a mystery (Widrow 2002). A large-scale primordial field is hard to maintain in a young galaxy because the galaxy rotates differentially, so that field lines get strongly wound up during galaxy evolution, while observations show significant pitch angles. "Seed" fields could also originate from the time of cosmological structure formation by the Weibel instability in shocks (Lazar et al. 2009) or could have been injected by the first stars or jets generated by the first black holes (Rees 2005), followed by a mechanism to amplify and organize the magnetic field.

The most promising mechanism to sustain magnetic fields in the interstellar medium of galaxies is the dynamo (Beck et al. 1996). In young galaxies without ordered rotation a small-scale dynamo (Brandenburg et al. 2005) possibly amplified the seed fields from the protogalactic phase to the energy density level of turbulence within less than $10^{9} \mathrm{yr}$. To explain the generation of large-scale fields in galaxies, the mean-field dynamo has been developed. It is based on turbulence, differential rotation and helical gas flows ( $\alpha$ effect), generated by supernova explosions (Gressel et al. 2008) or by cosmic-ray driven Parker loops (Hanasz et al. 2009). The mean-field dynamo in galaxy disks predicts that within a few $10^{9} \mathrm{yr}$ large-scale regular fields are excited from the seed fields (Arshakian et al. 2009, 2010), forming patterns ("modes") with different azimuthal symmetry in the disk and vertical symmetry in the halo.

The mean-field dynamo generates large-scale helicity with a non-zero mean in each hemisphere. As total helicity is a conserved quantity, the dynamo is quenched by the small-scale fields with opposite helicity unless these are removed from the system (Shukurov et al. 2006). Outflows are essential for an effective mean-field dynamo.

^ This work was supported by the European Commission Framework Program 6, Project SKADS, Square Kilometre Array Design Studies (SKADS), contract no 011938.
The magnetic fields in the intracluster medium could be seeded by outflows from starburst galaxies (Donnert et al: 2008) or from AGNs and amplified by turbulent wakes, cluster mergers or a turbulent dynamo (Subramanian et al. 2006; Ryu et al. 2008).

The fundamental questions are:

- When were the first magnetic fields generated: in young galaxies, in protogalactic clouds, or are they relics from the early Universe before the galaxies were formed?

- How and how fast were magnetic fields amplified in the interstellar and intracluster media?

- Did magnetic fields affect the evolution of galaxies and galaxy clusters?

- How important are magnetic fields for the physics of galaxies, like the efficiency to form stars from gas, the formation of spiral arms or the generation of gas outflows?

- How important are magnetic fields for the physics of the in tracluster medium, like cosmic-ray transport or anisotropic heat conduction?

- How strong and how ordered are magnetic fields in intergalactic space?

\section{Measuring magnetic fields}

The intensity of synchrotron emission is a measure of the number density of cosmic-ray electrons in the relevant energy range and of the strength of the total magnetic field component in the sky plane. Polarized emission emerges from ordered fields. As polarization "vectors" are ambiguous by $180^{\circ}$, they cannot distinguish regular (coherent) fields with a constant direction within the telescope beam from anisotropic fields which are generated from turbulent magnetic fields by compressing or shearing gas flows and frequently reverse their direction on small scales. Unpolarized synchrotron emission indicates turbulent fields with random directions which have been tangled or generated by turbulent gas flows. Only regular fields can give rise to Faraday rotation, while anisotropic and ran- 


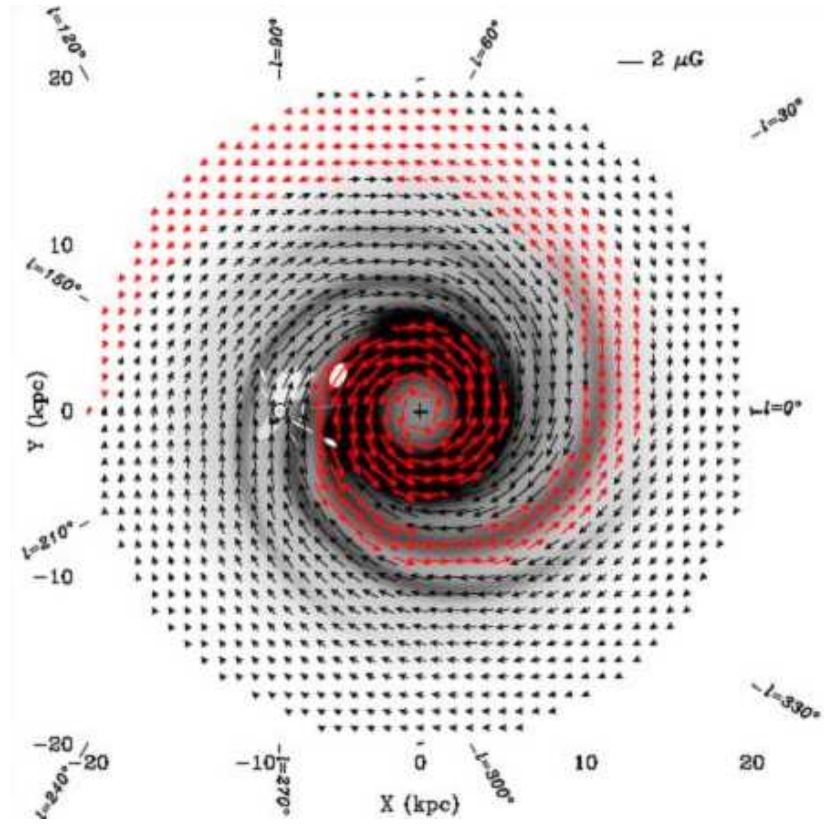

Fig. 1: The axisymmetric model of the large-scale structure of magnetic fields in the Milky Way disk, derived from polarization surveys and rotation measures of extragalactic sources (from Sun et al. 2008).

dom fields do not. Measurements of the Faraday rotation from multi-wavelength observations allow to determine the strength and direction of the regular field component along the line of sight.

A grid of Faraday rotation measures (RM) measurements towards polarized background sources with small angular sizes is hardly affected by Faraday depolarization within the foreground and a powerful tool to study magnetic field patterns in galaxies (Stepanov et al. 2008) and in clusters (Krause et al. 2009). A large number of background sources is required to recognize the field patterns, to separate the Galactic foreground contribution and to account for the intrinsic RM of extragalactic sources.

The method of Fourier transform of multi-channel spectropolarimetric data into RM space by RM Synthesis (Brentjens $\&$ de Bruyn 2005) is going to revolutionize radio polarization observations. It is able to separate RM components from distinct foreground and background regions and hence in principle to measure the 3-D structure of the magnetized interstellar medium in galaxies. If the medium has a relatively simple structure, e.g. a few emitting and Faraday-rotating regions, Faraday tomography will become possible. The distribution of the frequency channels across the total band and the channel width of the observation defines the Rotation Measure Spread Function (RMSF) (Heald 2009). Cleaning of the data cube with help of the known RMSF ("dirty beam") is similar to cleaning of synthesis data (Heald et al. 2009).

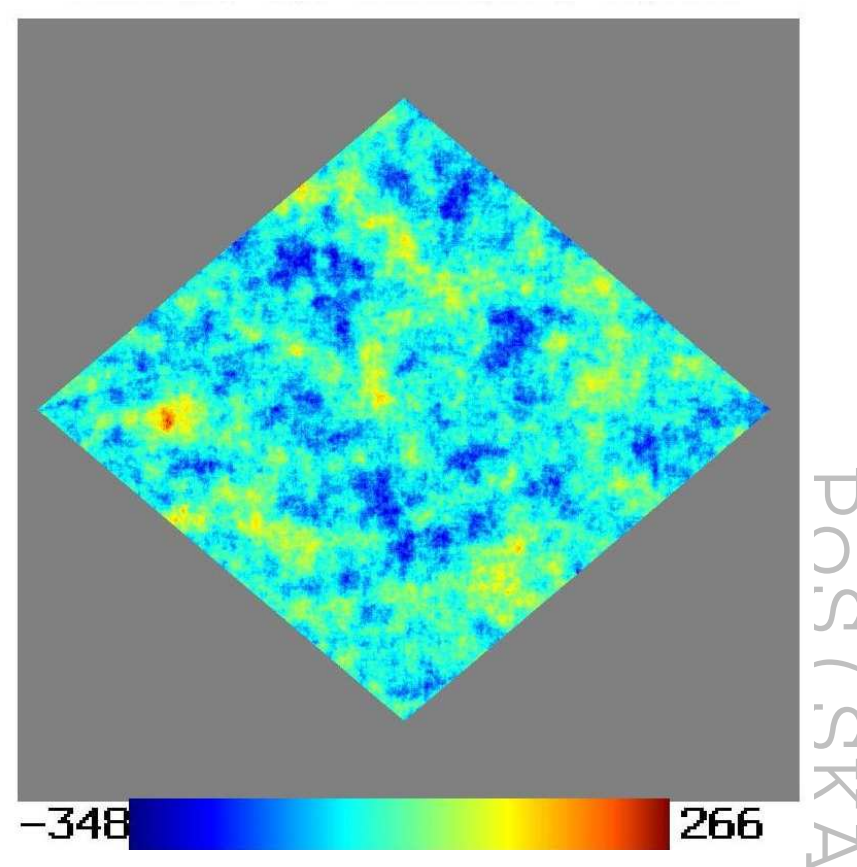

Fig. 2: Simulation of Faraday rotation measures at 1.6" resolution in a field of about $1.5^{\circ} \times 1.5^{\circ}$ at $1=130^{\circ}, \mathrm{b}=1^{\circ}$. The mean $\mathrm{RM}$ is $-86 \mathrm{rad}$ $\mathrm{m}^{-2}$, the RM dispersion $63 \mathrm{rad} \mathrm{m}^{-2}$ and the structure function has a $\Omega$ slope of about 0.7 (from Sun \& Reich 2009).

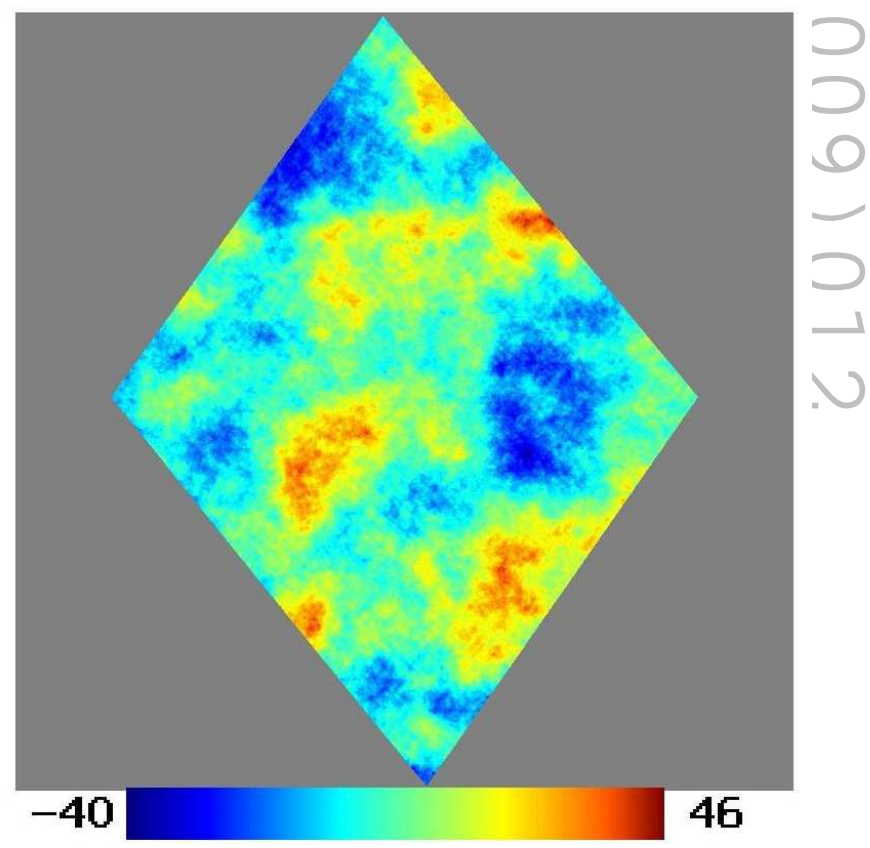

Fig. 3: Simulation of Faraday rotation measures at 1.6" resolution in a field of about $1.5^{\circ} \times 1.5^{\circ}$ at $1=138^{\circ}, \mathrm{b}=+70^{\circ}$. The mean $\mathrm{RM}$ is $+3 \mathrm{rad}$ $\mathrm{m}^{-2}$, the RM dispersion $13 \mathrm{rad} \mathrm{m}^{-2}$ and the structure function has a slope of about 0.9 (from Sun \& Reich 2009). 


\section{Magnetic fields in the Milky Way}

Sun et al. (2008) used the all-sky maps of polarized synchrotron emission at $1.4 \mathrm{GHz}$ from the Milky Way (Wolleben et al. 2006; Testori et al. 2008) and at 22.8 GHz from WMAP (Page et al. 2007) and the new Effelsberg Faraday RM survey of polarized extragalactic sources (Han et al., in prep.) were used to model the large-scale Galactic field, as part of the SKADS science simulations (Fig. 1). A large-scale reversal is required about 1-2 kpc inside the solar radius, which also agrees with the detailed study of RMs from extragalactic sources near the Galactic plane (Brown et al. 2007). More large-scale reversals may exist (Han et al. 2006). The local field is symmetric with respect to the Galactic plane, while the toroidal component of the halo field is probably antisymmetric. Models of a simple large-scale field structure of the Milky Way (e.g. by Han et al. 2002) could not be confirmed by statistical tests (Men et al. 2008). Similar to external galaxies, the Milky Way's regular field probably has a complex structure which can only be revealed by a larger sample of RM data from pulsars and extragalactic sources. The RM sample derived from the NVSS includes more than 37000 polarized sources, but it is based on only two channels with a small frequency separation (Taylor et al. 2009).

Detailed SKADS simulations of the diffuse Galactic emission towards various Galactic directions were performed by Sun \& Reich (2009). The turbulent field was assumed to be of Kolmogorov type with an inner scale of $0.025 \mathrm{pc}$ and and outer one of 10 pc. The fields near the Galactic plane (Fig. 2) and at high Galactic latitude (Fig. 3) are different in mean RM, RM dispersion and the slope of their structure functions. These simulations will help to estimate the Galactic foreground contribution and depolarization of extragalactic RM measurements with the SKA, and are also useful for the foreground subtraction of experiments to detect signals from the Epoch of Reionization.

\section{Magnetic fields in spiral galaxies}

The ordered (regular and/or anisotropic) fields traced by the polarized synchrotron emission are generally strongest (10$15 \mu \mathrm{G})$ in the regions between the optical spiral arms and oriented parallel to the adjacent spiral arms, in some galaxies forming magnetic arms. These are probably generated by a large-scale dynamo (Beck et al. 1996). In galaxies with strong density waves some of the ordered field is concentrated on the inner edge of the spiral arms (Fig. 4). The ordered magnetic field forms spiral patterns in almost every galaxy (Beck 2005).

Spiral fields can be generated by compression at the inner edge of spiral arms, by shear in interarm regions, or by dynamo action. Large-scale patterns of Faraday rotation measures (RM) are signatures of coherent dynamo fields and can be identified from polarized emission of the galaxy disks (Krause 1990) or from RM data of polarized background sources (Stepanov et al. 2008). The Andromeda galaxy M 31 hosts a dominating axisymmetric disk field (Fletcher et al. 2004), as predicted by dynamo models. Other candidates for a dominating axisymmetric disk field are the nearby spiral IC 342 (Krause et al. 1989) and the irregular Large Magellanic Cloud (LMC) (Gaensler

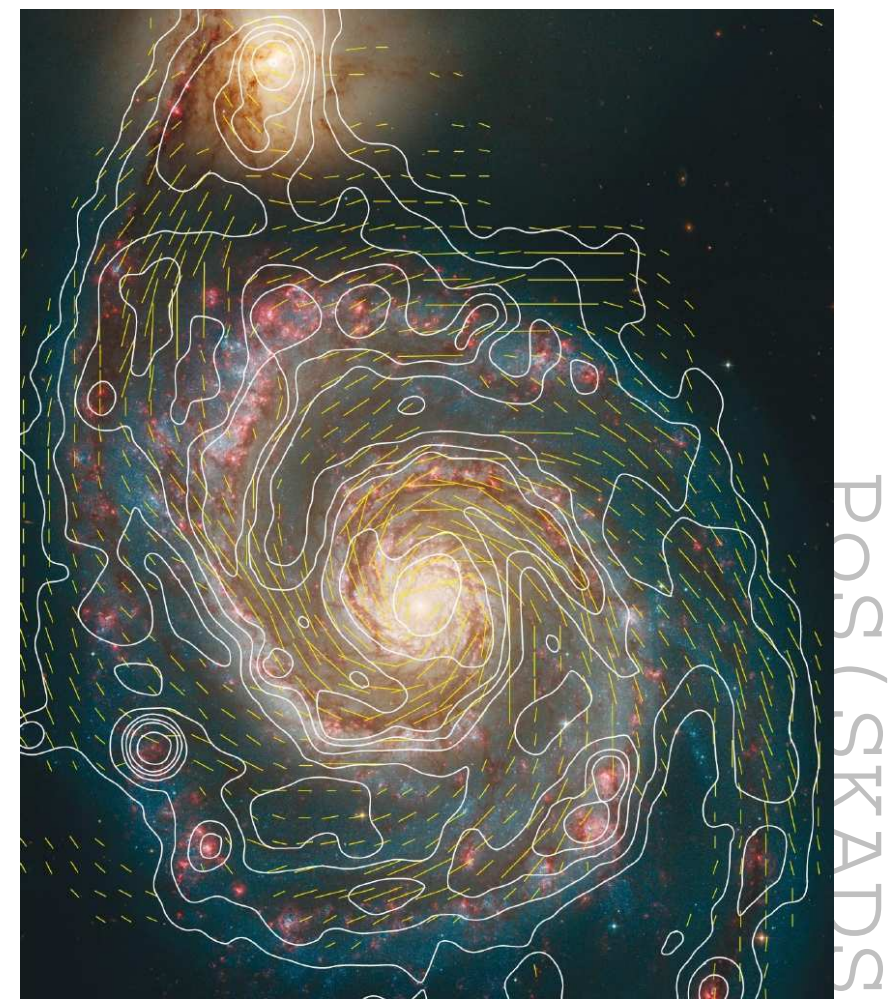

Fig. 4: Total radio emission (contours) and $B$-vectors of M 51, combined from observations at $6 \mathrm{~cm}$ wavelength with the VLA and Effelsberg telescopes and smoothed to 15 " resolution , overlaid onto an optical image from the HST. Copyright: MPIfR Bonn and Hubble Heritage Team. Graphics: Sterne und Weltraum (from Fletcher et al. 2010).

et al. 2005). However, in many observed galaxy disks no clear patterns of Faraday rotation were found. Either the field structure cannot be resolved with present-day telescopes or the timescale for the generation of large-scale modes is longer than the galaxy's lifetime (Arshakian et al. 2009).

Nearby galaxies seen edge-on generally show a diskparallel field near the disk plane. High-sensitivity observations of edge-on galaxies like NGC 891 (Fig. 5) and NGC 253 (Heesen et al. 2009) revealed vertical field components in the halo forming an "X-shaped" pattern. The field is probably transported from the disk into the halo by an outflow emerging from the disk.

As another part of the SKADS science simulations, dynamo theory was used to derive the timescales of amplification and ordering of magnetic fields in galaxies. Based on models describing the formation and evolution of dwarf and disk galaxies, an evolutionary model of turbulent and regular magnetic fields was developed that can be tested observationally (Arshakian et al. 2009, 2010):

- Strong turbulent fields (unpolarized synchrotron emission) can be observed in galaxies at $z<10$.

- Strong regular (coherent) fields (polarized synchrotron emission and RM) can be observed in Milky Way-type galaxies at $z \leq 3$. 


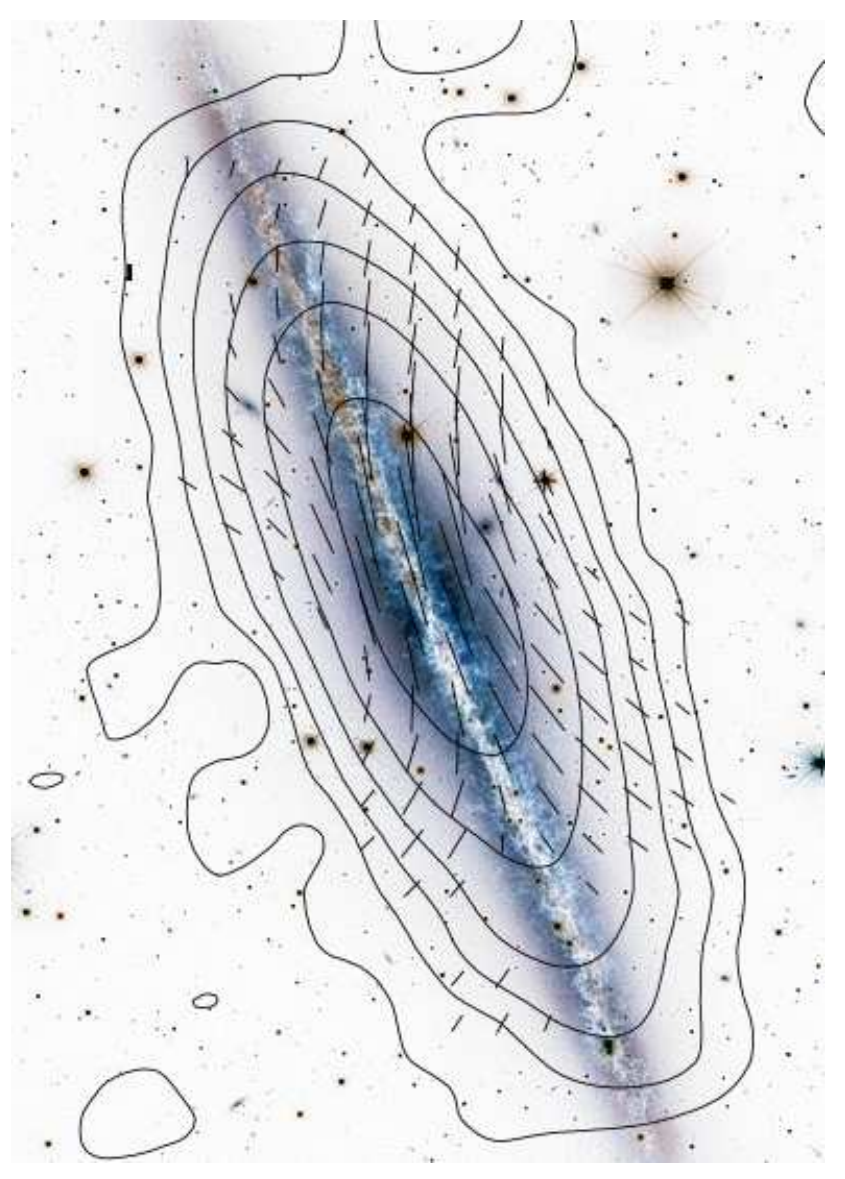

Fig. 5: Total radio emission (84" resolution) and B-vectors of the edge-on spiral galaxy NGC 891, observed at $3.6 \mathrm{~cm}$ wavelength with the Effelsberg $100 \mathrm{~m}$ telescope. The background optical image is from the CFHT. Copyright: MPIfR Bonn and CFHT/Coelum (from Krause 2009).

- Large-scale patterns of fully coherent regular fields (polarized synchrotron emission and large-scale RM patterns) can be observed in dwarf and Milky-Way type galaxies at $z \leq 1$.

- Giant galaxies (disk radius $>15 \mathrm{kpc}$ ) may not have generated fully coherent fields.

- Major mergers enhance turbulent fields but destroy regular fields and thus delay the formation of fully coherent fields.

The derived timescales of amplification of amplitude and ordering of the regular field and the sizes and star-formation rates of disk galaxies from the SKADS galaxy simulations (Obreschkow et al. 2009) were used to simulate the evolution of total radio intensity, polarization and Faraday rotation with age of a galaxy $(z \leq 3)$ at frequencies from $150 \mathrm{MHz}$ to $18 \mathrm{GHz}$ (Fig. 6).

About $10 \mathrm{RM}$ values in the area of a foreground galaxy are already sufficient to recognize a simple large-scale field structure (if the Galactic foreground can be properly subtracted), while more than 1000 values are required for a 3-D field reconstruction (Stepanov et al. 2008).

Ordered fields of nearby galaxies seen edge-on near the disk plane are preferably oriented parallel to the plane (Krause
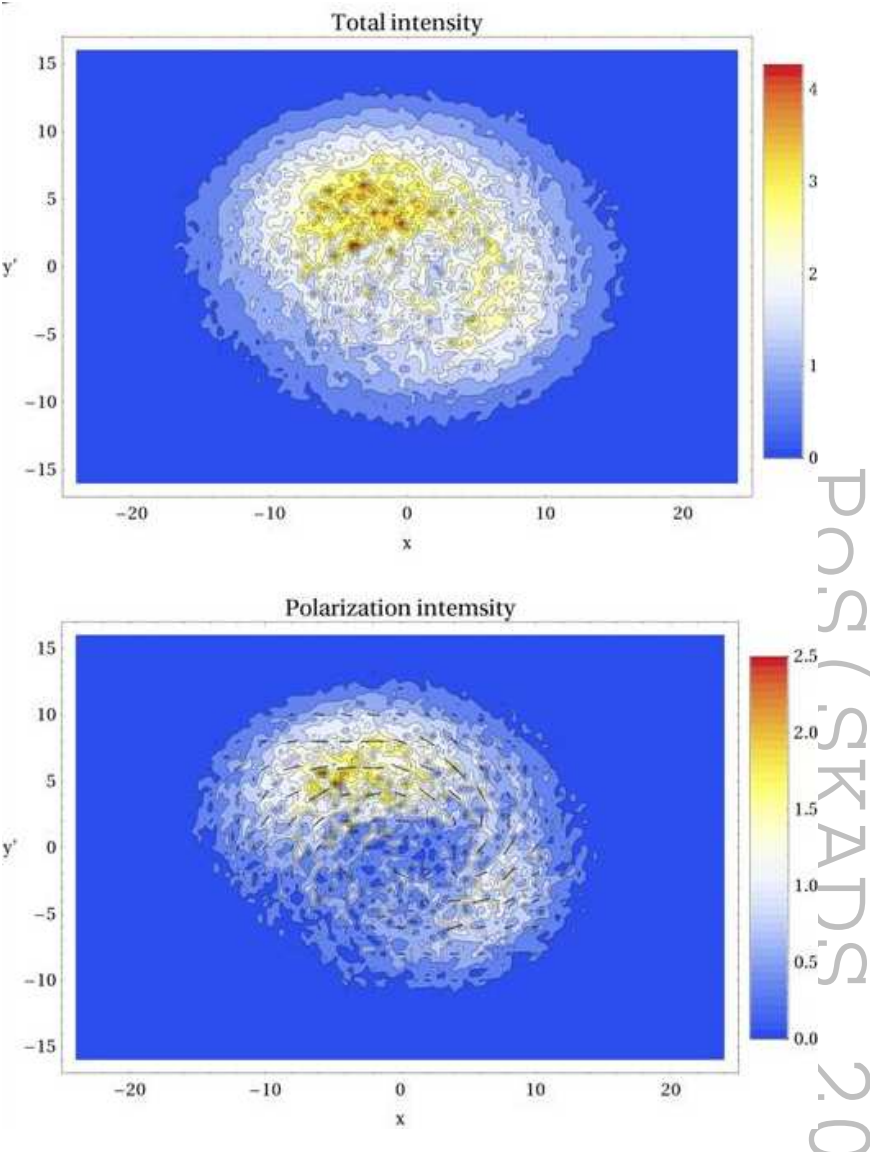

Fig. 6: Simulated map of the $5 \mathrm{GHz}$ radio emission from a spiral galaxy of $5 \mathrm{Gyr}$ age. Top: total intensity, bottom: polarized intensity (from Arshakian et al. in prep.).

2009). Polarized emission can be detected from unresolved galaxies if the inclination is larger than about $20^{\circ}$ (Stil et al. 2009a). This opens a new method to search for ordered fields in distant galaxies.

\section{Galaxy clusters}

Some fraction of galaxy clusters, mostly the X-ray bright ones, has diffuse radio emission (Cassano et al. 2008), emerging from diffuse halos and steep-spectrum relics (Fig. 7). Radio halos are mostly unpolarized and emerge from turbulent intracluster magnetic fields, observed as dispersion in RM decreasing with distance from the cluster center (Clarke et al. 2001). Relics can emit highly polarized radio waves from anisotropic magnetic fields generated by compression in merger shocks (Enßlin et al. 1998). A polarized region of about $1 \mathrm{Mpc}$ size was discovered in Abell 2255 (Govoni et al. 2005).

Equipartition strengths of the total magnetic field range from 0.1 to $1 \mu \mathrm{G}$ in halos and are higher in relics. On the other hand, Faraday rotation data towards background sources behind cluster halos reveals fields of a few $\mu \mathrm{G}$ strength fluctuating on coherence scales of a few kpc (Govoni \& Feretti 2004) and even $40 \mu \mathrm{G}$ in the cores of cooling flow clusters (Carilli $\&$ Taylor 2002) where they may be dynamically important. 


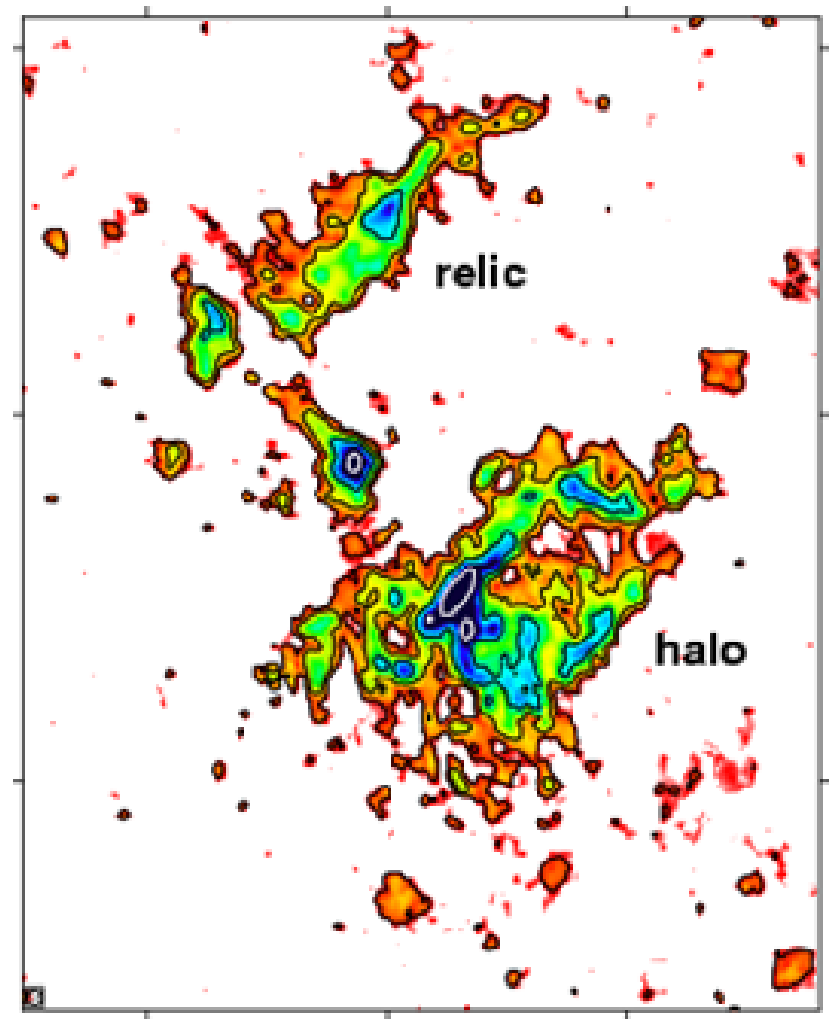

Fig. 7: Radio emission at $1.4 \mathrm{GHz}$ from the cluster A 2255. The field size is about 20' x 25' (from Govoni et al. 2005).

The reason for the discrepancy in field strengths is still under discussion.

High-resolution RM maps of radio galaxies embedded in a cluster allowed to derive the power spectra of the turbulent intracluster magnetic fields which are of Kolmogorov type and have coherence scales of about 1-5 kpc (Vogt \& Enßlin 2003).

Within the SKADS simulation project, Krause (2009) derived RM maps expected for a cluster population from an analytical cosmological model (Fig. 8). About $12 \%$ of the sky is covered with regions of $|R M|>10 \mathrm{rad} \mathrm{m}^{-2}$, and $66 \%$ of all clusters are located at $z \leq 1$. From this model and a model for the number density of polarized background sources, the number of background sources per cluster to be observed with the SKA Aperture Array was computed (Fig. 9). The number of RMs for clusters out to $\mathrm{z}=1$ will be sufficiently high to map the magnetic field distribution. At larger redshifts deeper integration will be needed. The total number of detectable clusters is about $10^{4}$ per field of view and per hour of integration. Measurement of the decrease of the maximum cluster RM with redshift can be used to constrain the magnetic field evolution $B \propto(1+z)^{n}$ within an accuracy of $\Delta n<0.4$.

\section{Intergalactic filaments}

The prediction of a large-scale "Cosmic Web" is one of the defining characteristics of large-scale structure simulations. Moreover, galaxies and the intra-cluster medium account for only approximately one third of the baryon density in the local

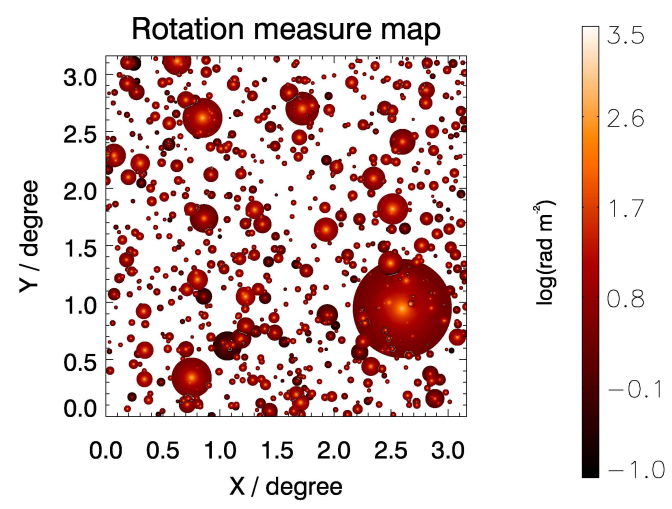

Fig. 8: Simulation of RMs from a cosmological cluster model (from Krause et al. 2009).

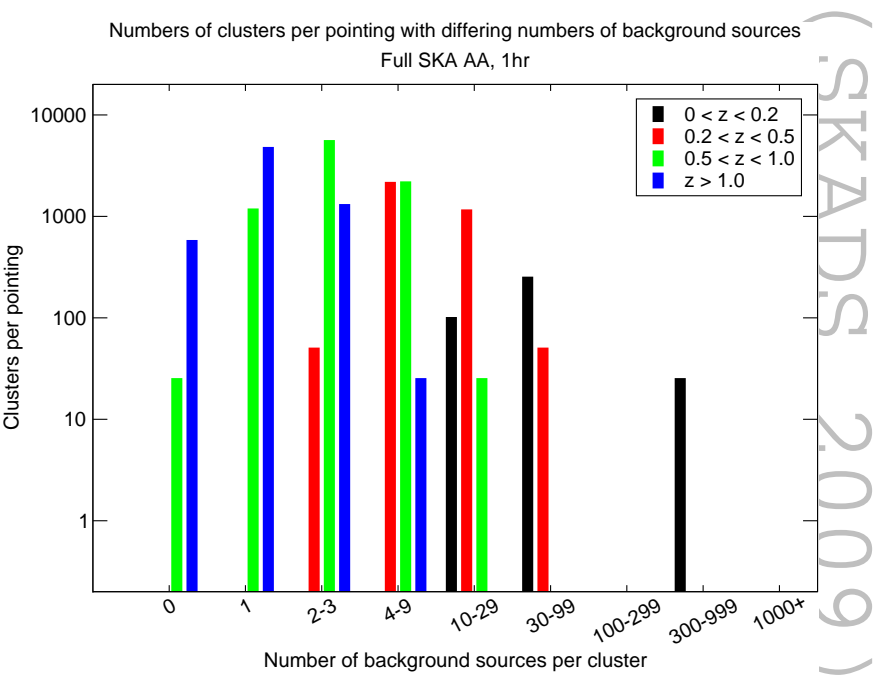

Fig. 9: Simulation of the number of RMs per cluster observable with the SKA Aperture Array (300-1000 MHz) per field of view (250 deg ${ }^{2}$ ) within $1 \mathrm{~h}$ integration time (Krause et al. 2009).

Universe expected in a concordance cosmology. The majority of the missing baryons are likely to reside in a warm-hot intergalactic medium (WHIM) which in turn is expected to reside in the cosmic web of the large-scale structure. Galaxy clusters, the brightest knots of the Cosmic Web, are filled with relativistic particles and magnetic fields, as observed through the radio synchrotron emission.

The search for magnetic fields in the intergalactic medium (IGM) is of fundamental importance for cosmology. All "empty" space in the Universe may be magnetized. Its role as the likely seed field for galaxies and clusters and its possible relation to structure formation in the early Universe, places considerable importance on its discovery. Models of structure formation predict strong intergalactic shocks which enhance the field.

Various mechanisms have been suggested for the origin of a magnetic field in the cosmic web. The field could be produced via the Weibel instability (a small-scale plasma instability) at structure formation shocks (Medvedev et al. 2004). Another 
possibility is that the field is injected into the WHIM via the action of injection from galactic black holes (AGNs) and other outflows. In each case the field is subsequently amplified by compression and large-scale shear-flows (Brüggen et al. 2005). Ryu et al. (2008) have argued that highly efficient amplification is possible via MHD turbulence, with the source of the turbulent energy being the structure formation shocks themselves (Fig. 14). Estimates of the strength of the turbulent field in filaments obtained from MHD simulations with a primordial seed field range typically between $0.1 \mu \mathrm{G}$ and $0.01 \mu \mathrm{G}$, while regular fields are weaker.

To date there has been no detection of a general magnetic field in the IGM. In an intergalactic region of about $2^{\circ}$ extent west of the Coma Cluster, containing a group of radio galaxies, enhanced synchrotron emission yields an equipartition total field strength of $0.2-0.4 \mu \mathrm{G}$ (Kronberg et al. 2007). Xu et al. (2006) observed an excess of rotation measures (RM) towards two super-clusters which may indicate regular magnetic fields of $<0.3 \mu \mathrm{G}$ on scales of order $500 \mathrm{kpc}$. Lee et al. (2009) found a statistical correlation at the $4 \sigma$ level of the RMs of background sources with the galaxy density field and derived a $30 \mathrm{nG}$ intergalactic field with about $1 \mathrm{Mpc}$ coherence length.

\section{Prospects}

Future radio telescopes will widen the range of observable magnetic phenomena. High-resolution, deep observations at high frequencies, where Faraday effects are small, require a major increase in sensitivity for continuum observations which will be achieved by the Extended Very Large Array (EVLA) and the planned Square Kilometre Array (SKA). The detailed structure of the magnetic fields in the ISM of galaxies, in galaxy halos, in cluster halos and in cluster relics can then be observed. The turbulence power spectra of the magnetic fields can be measured (Vogt \& Enßlin 2003). Direct insight into the interaction between gas and magnetic fields in these objects will become possible. The SKA will also allow to measure the Zeeman effect in much weaker magnetic fields in the Milky Way and in nearby galaxies.

Detection of polarized emission from distant, unresolved galaxies indicates large-scale ordered fields (Stil et al. 2009a), and statistics can be compared with the predictions of dynamo theory (Arshakian et al. 2009). The SKA will detect MilkyWay type galaxies at $z \leq 1.5$ (Fig. 10) and their polarized emission at $z \leq 0.5$ (assuming $10 \%$ percentage polarization). Bright starburst galaxies can be observed at larger redshifts, but are not expected to host ordered or regular fields. Cluster relics are also detectable at large redshifts through their integrated polarized emission. This effect still has to be investigated.

Unpolarized synchrotron emission, signature of turbulent magnetic fields, can be detected with the SKA out to very large redshifts for starburst galaxies, depending on luminosity and magnetic field strength (Fig. 10), and also for cluster halos. However, for fields weaker than $3.25 \mu \mathrm{G}(1+z)^{2}$, energy loss of cosmic-ray electrons is dominated by the inverse Compton effect with CMB photons, so that their energy appears mostly in X-rays and not in the radio range. On the other hand, for strong fields the energy range of the electrons emit-

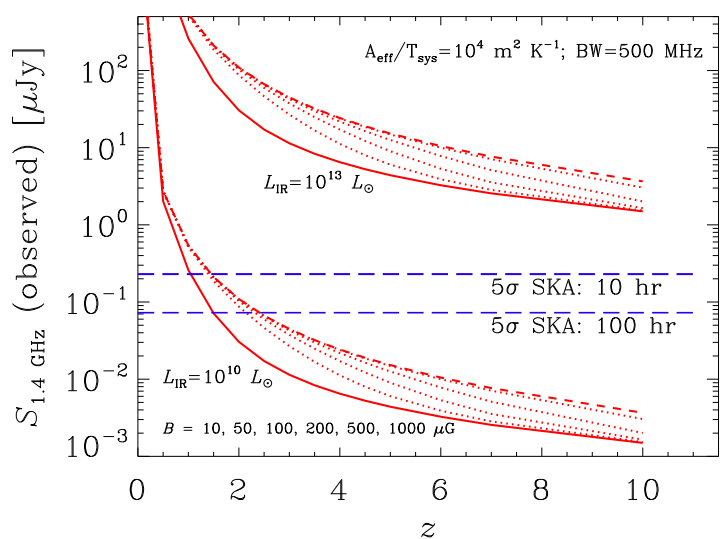

Fig. 10: Synchrotron emission at $1.4 \mathrm{GHz}$ as a function of redshift $z$ and magnetic field strength $B$, and the $5 \sigma$ detection limits for $10 \mathrm{~h}$ and $100 \mathrm{~h}$ with the SKA (from Murphy 2009).

ting at a $1.4 \mathrm{GHz}$ drops to low energies, where ionization and bremsstrahlung losses may become dominant. In summary, the mere detection of synchrotron emission at high redshifts will constrain the range of allowed magnetic field strengths.

Forthcoming low-frequency radio telescopes like the Low Frequency Array (LOFAR), Murchison Widefield Array (MWA), Long Wavelength Array (LWA) and the lowfrequency SKA will be suitable instruments to search for extended synchrotron radiation at the lowest possible levels in outer galaxy disks and the transition to intergalactic space (Beck 2009) and in steep-spectrum cluster halos (Brunetti et al. 2008).

If polarized emission is too weak to be detected, the method of RM grids towards background QSOs can still be applied. Here, the distance limit is given by the polarized flux of the background QSO which can be much higher than that of the intervening galaxy. A reliable model for the global structure of the magnetic field of nearby galaxies needs a large number of RM values from a large number density of polarized background sources, hence large sensitivity and/or high survey speed. The "POSSUM-Wide" survey at $1.4 \mathrm{GHz}$ with the planned Australia SKA Pathfinder (ASKAP) telescope with $30 \mathrm{deg}^{2}$ field of view (Gaensler et al. 2010) will measure about $100 \mathrm{RM}$ values from polarized extragalactic sources per square degree within $10 \mathrm{~h}$ integration time, assuming a slope of the cumulative source count function of $\gamma=-0.9$ (Stepanov et al. 2008). Similarly deep integrations with the EVLA and with MeerKAT will show about 5 times more sources, but their fields of view are small and will allow only a limited number of pointings.

The SKA pulsar survey will find about 20000 new pulsars which will be mostly polarized and reveal RMs (Fig. 11), perfectly suited to measure the Milky Way's magnetic field with extremely high precision.

The SKA "Magnetism" Key Science Project plans to observe a wide-field survey (at least $10^{4} \mathrm{deg}^{2}$ ) around $1 \mathrm{GHz}$ with $1 \mathrm{~h}$ integration per field which will be able to detect sources 


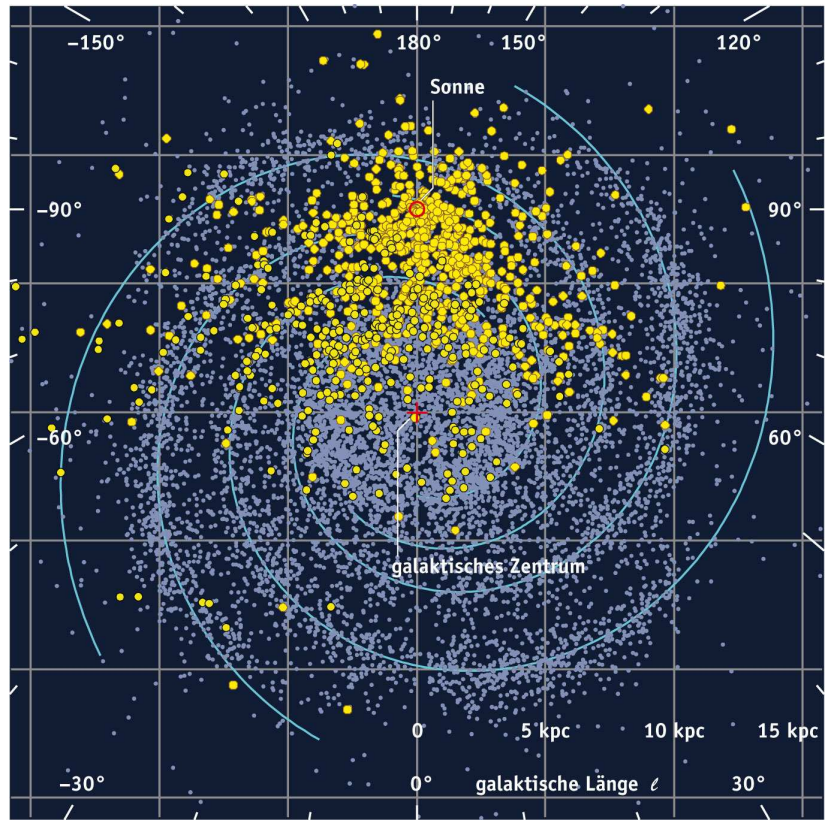

Fig. 11: Simulation of about 20000 new pulsars (blue) in the Milky Way that will be detected with the SKA, compared to about 2000 pulsars known today (yellow). Graphics: Sterne und Weltraum (from Cordes, priv. comm).

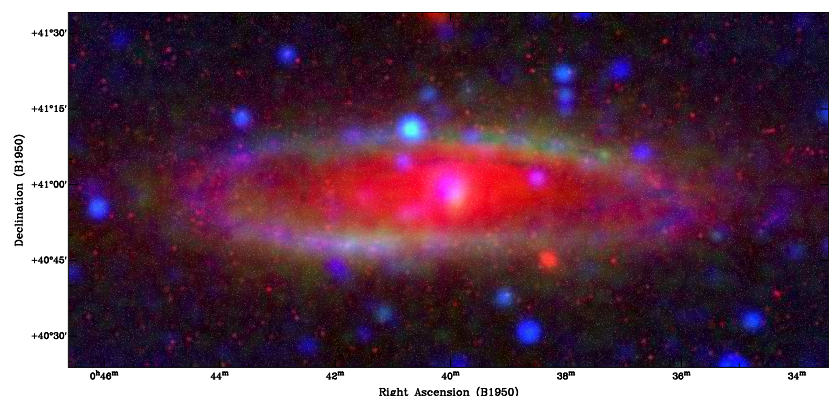

Fig. 12: Simulation of the number of RMs (about 10000 ) in the region of M 31 observable with the SKA within $1 \mathrm{~h}$ integration time (from Gaensler, priv. comm.).

with $0.5-1 \mu \mathrm{Jy}$ flux density and measure at least $1500 \mathrm{RMs}$ per square degree. This will contain at least $2 \times 10^{7} \mathrm{RMs}$ from compact polarized extragalactic sources at a mean spacing of $\simeq 90^{\prime \prime}$, plus at least $10^{4} \mathrm{RM}$ values from pulsars with a mean spacing of $\simeq 30^{\prime}$ (Gaensler et al. 2004, 2009). If the cumulative source count function of polarized sources has a slope as steep as $\gamma=-1.1$ (Stepanov et al. 2008), the total RM number will be $10 \times$ larger and the mean spacing as low as $\simeq 30^{\prime \prime}$. More than $10000 \mathrm{RM}$ values are expected in the area of M 31 (Fig. 12) and will allow the detailed reconstruction of the 3-D field structure in this and many other nearby galaxies, while simple patterns of regular fields can be recognized out to distances of about $100 \mathrm{Mpc}$ (Stepanov et al. 2008) where the polarized flux is too low to be mapped. The magnetism of cluster halos can be measured by the RM grid to redshifts of about 1 (Fig. 9).

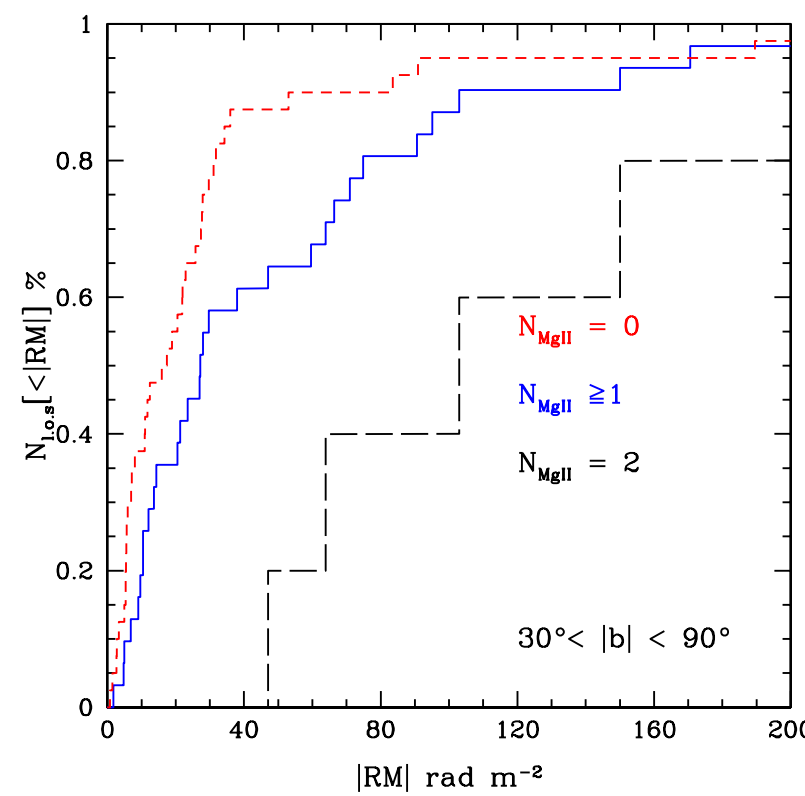

Fig. 13: Cumulative RM distributions for 31 sightlines towards QSOS with optical spectra showing one or two strong MgII absorption line systems (thick blue line), for 5 sightlines with two strong MgII systems (black dashed line) and for 40 sightlines without strong MgII absorption lines (thin red dashed line). The redshifts of the intervening galaxies are between $\mathrm{z}=0.6$ and 2 (from Bernet et al. 2008).

The SKA "Magnetism" Key Science Project also plans a deep-field survey (at least $40 \mathrm{deg}^{2}$ ) with $100 \mathrm{~h}$ integration per field (Stil et al. 2009b), to detect polarized sources with $50 \mathrm{nJy}$ flux density and measure their RMs. With at least $3 \times 10^{4} \mathrm{RMs}$ per square degree and at a mean spacing of 10-20", about 3 times larger distances can be reached than with the wide-field survey.

Faraday rotation in the direction of QSOs allows to determine the strength and pattern of a regular field in an intervening galaxy (Kronberg et al. 1992). Significant regular fields of several $\mu \mathrm{G}$ strength were detected in distant galaxies (Fig. 13). This method can be applied to distances of young QSOs $(z \simeq 5)$, and the SKA will provide a large data sample for excellent statistics. Mean-field dynamo theory predicts RMs from regular galactic fields at $z \leq 3$ (Arshakian et al. 2009), but the RM values are reduced by the redshift dilution factor of $(1+z)^{-2}$. If an overall IGM field with a coherence length of a few Mpc existed in the early Universe and its strength varied proportional to $(1+z)^{2}$ (Widrow 2002), its signature may become evident at redshifts of $z>3$. Averaging over a large number of RMs is required to see the IGM signal. Our goal is to detect an IGM magnetic field of $0.1 \mathrm{nG}$ at $5 \sigma$ in the presence of Galactic foregrounds. Kolatt (1998) has calculated that an $\mathrm{RM}$ density of $\approx 1000$ sources per $\mathrm{deg}^{-2}$ is sufficient to meet this requirement.

If the filaments of the local Cosmic Web outside clusters contain a magnetic field (Fig. 14), possibly enhanced by IGM shocks, we can hope to detect this field by direct observation of its total synchrotron emission (Keshet et al. 2004) and possi- 


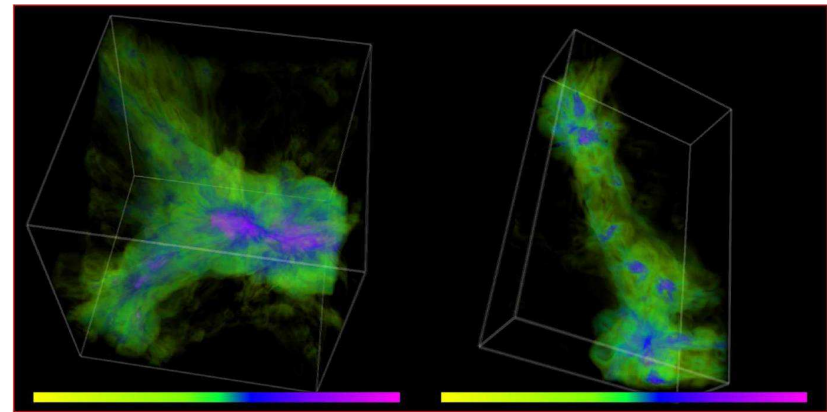

Fig. 14: Simulation of magnetic fields in the Cosmic Web (at $z=0$ ) in a volume of $25 h^{-1} \mathrm{Mpc}^{3}$ centered around a cluster complex (left panel), and in a volume of $25 \times 15.6 \times 6.25 \mathrm{~h}^{-1} \mathrm{Mpc}^{3}$ including a number of galaxy groups along a filament (right panel). The color codes the magnetic field strength (logarithmically scaled) from $0.1 \mathrm{nG}$ (yellow) to $10 \mu \mathrm{G}$ (magenta). Clusters and groups are shown with magenta and blue, while filaments are green (from Ryu et al. 2008).

bly its polarization, or by Faraday rotation towards background sources. For fields of $\approx 10^{-8}-10^{-7} \mathrm{G}$ with $1 \mathrm{Mpc}$ coherence length and $n_{e} \approx 10^{-5} \mathrm{~cm}^{-3}$ electron density, Faraday rotation measures between 0.1 and $1 \mathrm{rad} \mathrm{m}^{-2}$ are expected. A $30 \mathrm{nG}$ regular field on a coherence scale of $1 \mathrm{Mpc}$ (Lee et al. 2009) would generate about $0.2 \mathrm{rad} \mathrm{m}^{-2}$, which probably cannot be detected directly. Promising is a statistical analysis like the measurement of the power spectrum of the magnetic field of the Cosmic Web (Kolatt 1998) or the cross-correlation with other large-scale structure indicators like the galaxy density field. Detection of a general IGM field, or placing stringent upper limits on it, will provide powerful observational constraints on the origin of cosmic magnetism.

The SKA wide-field RM survey is planned in the frequency band of the SKA Aperture Array (300-1000 MHz). At lower frequencies synchrotron emission is stronger but depolarization effects are more severe. With a model of depolarization by internal Faraday dispersion, the wavelength of maximum polarized emission can be computed as a function of RM dispersion (Arshakian et al., in prep.). Polarized emission from galaxy disks should be observed around $1 \mathrm{GHz}$, while galaxy halos and intracluster media can be best observed between $300 \mathrm{MHz}$ and $1 \mathrm{GHz}$. A model of external depolarization of the emission from background sources in a foreground cluster leads to a similar result (Krause et al. 2007).

\section{Summary}

The SKA and its precursors will measure the structure and strength of the magnetic fields in the Milky Way, in intervening galaxies and clusters, and in the intergalactic medium. Looking back into time, the future telescopes can shed light on the origin and evolution of cosmic magnetic fields.

The observational methods are:

- 3-D RM grid from extragalactic sources and pulsars to map the detailed 3-D structure of the Milky Way's magnetic field
- High-resolution mapping of total and polarized synchrotron emission from nearby galaxies, clusters halos and relics

- Reconstruction of 3-D field patterns in nearby galaxies and nearby clusters from RMs towards polarized background sources

- Recognition of simple patterns of regular fields in galaxies from RMs towards polarized background sources (at $z \leq$ 0.02)

- Detection of polarized synchrotron emission from distant galaxies and distant halo relics (at $z \leq 0.5$ )

- Recognition of structure of turbulent fields in clusters from RMs towards polarized background sources (at $z \leq 0.5$ )

- Detection of total synchrotron emission from distant starburst galaxies and distant clusters (at $z \leq 3$ )

- Detection of regular fields in very distant intervening galaxies towards QSOs (at $z \leq 5$ ).

\section{References}

Arshakian, T. G., Beck, R., Krause, M. \& Sokoloff, D. 2009 A\&A, 494, 21

Arshakian, T.G., Stepanov, R., Beck, R., Krause, M., Sokoloff, D., Frick, P., "Exploring the magnetic fields in local and distant galaxies," 2010, in Proc. Wide Field Science and Technology for the SKA, Limelette, Belgium, 2 S.A. Torchinsky et al. (eds), ASTRON, ISBN 978-90805434-5-4

Beck, R. 2005, in Cosmic Magnetic Fields, ed. R. Wielebinski \& R. Beck (Springer, Berlin), 41

Beck, R. 2009, Rev. Mex. AyA, 36, 1

Beck, R., Brandenburg, A., Moss, D., Shukurov, A. \& Sokoloff, D. 1996, Ann. Rev. A\&A, 34, 155

Bernet, M. L., Miniati, F., Lilly, S. J., Kronberg, P.P. \& Dessauges-Zavadsky, M. 2008, Nature, 454, 302

Brandenburg, A. \& Subramanian, K. 2005, Phys. Rep., 417, 1

Brentjens, M. A. \& de Bruyn, A. G. 2005, A\&A, 441, 1217

Brown, J. C., Haverkorn, M., Gaensler, B. M., et al. 2007, ApJ, 663,258

Brüggen, M., Ruszkowski, M., Simionescu, A., Hoeft, M. \& Dalla Vecchia, C. 2005, ApJ, 631, L21

Brunetti, G., Giacintucci, S., Cassano, R., et al. 2008, Nature, 455,944

Carilli, C. L. \& Taylor, G. B. 2002, Ann. Rev. A\&A, 40, 319

Cassano, R., Brunetti, G., Venturi, T., et al. 2008, A\&A, 480, 687

Clarke, T.E., Kronberg, P. P. \& Böhringer, H. 2001, ApJ, 547, L111

Donnert, J., Dolag, K., Lesch, H. \& Müller, E. 2008, MNRAS, 392,1365

Enßlin, T. A., Biermann, P.L., Klein, U. \& Kohle, S. 1998, A\&A, 332, 395

Fletcher, A., Berkhuijsen, E. M., Beck, R. \& Shukurov, A. 2004, A\&, 414, 53

Fletcher, A., Beck, R., Shukurov, A., Berkhuijsen, E. M. \& Horellou, C. 2010, arXiv:1001.5230

Gaensler, B. M., Beck, R. \& Feretti, L. 2004, in Science with the Square Kilometer Array, ed. C. Carilli \& S. Rawlings (Elsevier, Amsterdam), New Astr. Rev., 48, 1003 
Gaensler, B. M., Haverkorn, M., Staveley-Smith, L., et al. 2005, Science, 307, 1610

Gaensler, B. M., Beck, R., Geisbüsch, J. \& Alexander, P. 2009, in The Square Kilometre Array Design Reference Mission, ed. SKA Science Working Group, 31

Gaensler, B. M., Landecker, T. L. \& Taylor, A. R. 2010, BAAS, 42,470

Govoni, F. \& Feretti, L. 2004, Int. J. Mod. Phys. D, 13, 1549

Govoni, F., Murgia, M., Feretti, L., Giovannini, G., Dallacasa, D. \& Taylor, G. B. 2005, A\&A, 430, L5

Gressel, O., Elstner, D., Ziegler, U. \& Rüdiger, G. 2008, A\&A, 486, L35

Han, J. L., Manchester, R. N., Lyne, A. G. \& Qiao, G. J. 2002, ApJ, 570, L17

Han, J. L., Manchester, R. N., Lyne, A. G., Qiao, G. J. \& van Straten, W. 2006, ApJ, 642, 868

Hanasz, M., Wóltański, D. \& Kowalik, K. 2009, ApJ, 706, L155

Heald, G. 2009, LOFAR Magnetism KSP Memo No. 1

Heald, G., Braun, R. \& Edmonds, R. 2009, A\&A, 503, 409

Heesen, V., Krause, M., Beck, R. \& Dettmar, R.-J. 2009, A\&A, 506, 1123

Keshet, U., Waxman, E. \& Loeb, A. 2004, in Science with the Square Kilometer Array, ed. C. Carilli \& S. Rawlings (Elsevier, Amsterdam), New Astr. Rev., 48, 1119

Kolatt, T. 1998, ApJ, 495, 564

Krause, Marita 1990, in Galactic and Intergalactic Magnetic Fields, ed. R. Beck, R. Wielebinski \& P.P. Kronberg (Dordrecht: Kluwer), 187

Krause, Marita 2009, Rev. Mex. AyA, 36, 25

Krause, Marita, Hummel, E. \& Beck, R. 1989, A\&A, 217, 4

Krause, Martin, Alexander, P. \& Bolton, R. 2007, in From Planets to Dark Energy: the Modern Radio Universe, Proc. of Science, 109

Krause, Martin, Alexander, P., Bolton, R., Geisbüsch, J., Green D. A. \& Riley, J. 2009, MNRAS, 400, 646

Kronberg, P. P., Perry, J. J. \& Zukowski, E. L.H. 1992, ApJ, 387,528

Kronberg, P. P., Kothes, R., Salter, C. J. \& Perillat, P. 2007, ApJ, 659,267

Lazar, M., Schlickeiser, R., Wielebinski, R. \& Poedts, S. 2009, ApJ, 693, 1133

Lee, J., Pen, U.-L., Taylor, A. R., Stil, J.M. \& Sunstrum, C. 2009, arXiV:0906.1631

Medvedev, M. V., Silva, L. O., Fiore, M., Fonseca, R. A. \& Mori, W. B. 2004, JKAS, 37, 533

Men, H., Ferrière, K. \& Han, J. L. 2008, A\&A, 486, 819

Murphy, E. 2009, ApJ, 706, 482

Obreschkow, D., Klöckner, H.-R., Heywood, I., Levrier, F. \& Rawlings, S. 2009, ApJ, 703, 1890

Page, L., Hinshaw, G., Komatsu, E., et al. 2007, ApJS, 170, 335

Rees, M.J. 2005, in Cosmic Magnetic Fields, ed. R. Wielebinski \& R. Beck (Springer, Berlin), 1

Ryu, D., Kang, H., Cho, J. \& Das, S. 2008, Science, 320, 909

Shukurov, A., Sokoloff, D., Subramanian, K. \& Brandenburg, A. 2006, A\&A, 448, L33

Stepanov, R., Arshakian, T. G., Beck, R., Frick, P. \& Krause, M. 2008, A\&A, 480, 45
Stil, J., Krause, M., Beck, R. \& Taylor, R. 2009a, ApJ, 693, 1392

Stil, J. M., Taylor, A. R., Beck, R., Gaensler, B. M., Geisbüsch, J. \& Alexander, P. 2009b, in The Square Kilometre Array Design Reference Mission, ed. SKA Science Working Group, 20

Subramanian, K., Shukurov, A. \& Haugen, N.E. L. 2006, MNRAS, 366, 1437

Sun, X. H. \& Reich, W. 2009, A\&A, 507, 1087

Sun, X.H., Reich, W., Waelkens, A. \& Enßlin, T. A. 2008, A\&A, 477, 573

Taylor, A. R., Stil, J. M. \& Sunstrum, C. 2009, ApJ, 702, 1230

Testori, J. C., Reich, P. \& Reich, W. 2008, A\&A, 484, 733

Vogt, C. \& Enßlin, T. A. 2003, A\&A, 412, 373

Widrow, L. M. 2002, Rev. Mod. Phys., 74, 775

Wolleben, M., Landecker, T.L., Reich, W. \& Wielebinski, R.? 2006, A\&A, 448, 411

Xu, Y., Kronberg, P. P., Habib, S. \& Dufton, Q. W. 2006, ApJ, 637, 19 